\title{
Numerical method for accessing the universal scaling function for a multiparticle discrete time asymmetric exclusion process
}

\author{
Nicholas Chia and Ralf Bundschuh* \\ Department of Physics, Ohio State University, 191 W. Woodruff Ave., Columbus, Ohio 43210, USA \\ (Received 8 March 2005; revised manuscript received 15 September 2005; published 7 November 2005)
}

\begin{abstract}
In the universality class of the one-dimensional Kardar-Parisi-Zhang (KPZ) surface growth, Derrida and Lebowitz conjectured the universality of not only the scaling exponents, but of an entire scaling function. Since and Derrida and Lebowitz's original publication [Phys. Rev. Lett. 80, 209 (1998)] this universality has been verified for a variety of continuous-time, periodic-boundary systems in the KPZ universality class. Here, we present a numerical method for directly examining the entire particle flux of the asymmetric exclusion process (ASEP), thus providing an alternative to more difficult cumulant ratios studies. Using this method, we find that the Derrida-Lebowitz scaling function (DLSF) properly characterizes the large-system-size limit $(N \rightarrow \infty)$ of a single-particle discrete time system, even in the case of very small system sizes $(N \leqslant 22)$. This fact allows us to not only verify that the DLSF properly characterizes multiple-particle discrete-time asymmetric exclusion processes, but also provides a way to numerically solve for quantities of interest, such as the particle hopping flux. This method can thus serve to further increase the ease and accessibility of studies involving even more challenging dynamics, such as the open-boundary ASEP.
\end{abstract}

DOI: 10.1103/PhysRevE.72.051102 PACS number(s): 05.40. $-\mathrm{a}, 02.50 .-\mathrm{r}, 82.20 .-\mathrm{w}$, 89.75.Da

\section{INTRODUCTION}

The Kardar-Parisi-Zhang (KPZ) equation [1] describes a rich variety of processes such as surface growth [2,3], directed polymers [4-6], and avalanches [7,8]. And accordingly, the massive array of literature (see [9-11] and references therein) on the topic of KPZ theory reflects the central role the KPZ equation plays in the study of stochastic dynamic processes. The wide variety of dynamic processes governed by the KPZ equation form the so-called KPZ universality class - a class of seemingly unrelated dynamics whose bulk properties obey, on a coarse-grained level, this one master equation. One member of the KPZ universality class, the well-studied one-dimensional asymmetric exclusion process (ASEP), describes a driven lattice gas with hard-core exclusions [12] and has also been applied to studies of highway traffic $[13,14]$, protein synthesis [15], and sequence alignment [16-18]. Characterizing the underlying properties of the ASEP promises a greater understanding for these specific studies as well as insights into the broader KPZ universality class. Not surprisingly, much effort has been spent calculating some of the many properties of the ASEP such as the density profile, steady states, mass gaps, and diffusion constants [19-28]. Though many questions still remain, these myriad studies have helped to uncover a great deal of insight into the ASEP.

In their study of the ASEP, Derrida and Lebowitz [29] extended the Bethe ansatz approach of Gwa and Spohn [23] in order to solve the totally ASEP for particle displacement in the asymptotic limit of large system size [29]. One of the most interesting aspects of their solution involves the scaling function $G$, describing the nonlinear behavior of the total particle flux. The Derrida-Lebowitz scaling function (DLSF)

*Electronic address: bundschuh@mps.ohio-state.edu is independent of any model parameters. Thus, this scaling function was conjectured to be universal-i.e., characteristic of all KPZ systems. In a follow-up study, Derrida and Appert analytically continued the DLSF, successfully completing the solution for all space in the asymptotic limit of large system sizes [30]. Since then, a large number of studies have given strong evidence for the universality of the DLSF. These studies fall into two classes. On the one hand, for a few closely related variants of the continuous-time totally ASEP $[4,7,31]$ the characteristic DLSF behavior has been analytically verified. On the other hand, numerical studies have bolstered the universality claim of the DLSF [7,30-33] for a much broader range of systems including genuinely discrete time systems. However, these numerical methods do not directly verify the universality of the DLSF, but rather verify the universality of certain cumulant ratios which must be universal if the DLSF is universal [30]. These numerical approaches cannot directly verify the DLSF since they use sampling methods that are inherently unable to probe the full DLSF which contains information about statistically rare events. In addition to that, both analytical and numerical studies rely on examining the behavior of the so-called scaling region [30] which applies only in the limit of very large system sizes $(\sim 1280)$, especially in the discrete-time case $(>10240)$.

By examining properties applicable to the intermediatescaling region, we created a method for directly measuring the DLSF for discrete-time systems of considerably smaller size $(<22)$. Our method works without resorting to the stochastic sampling that makes cumulant methods so time consuming. As an application, we show that the discrete-time ASEP with both single and multiple particles per site under any parameter choice is characterized by the same DLSF conjectured to be universal for all processes within the KPZ universality class. Our method can also be used to study the particle hopping in other ASEP scenarios, including the open-boundary ASEP. Once DLSF behavior has been veri- 


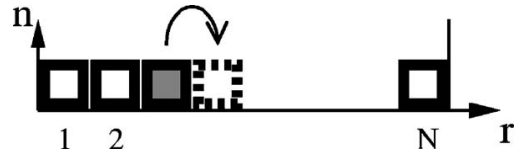

FIG. 1. Diagram of a periodically bound totally ASEP. Only the particle shaded in gray, on site 3, may move to the space on the right, outlined by a dotted line, with a probability $d t$. The remaining particles are prevented from moving by an adjacent particle occupying the space to the immediate right. More explicitly, the particle on site 2 finds its advance blocked by the particle that resides on site 3. Meanwhile, the particle on site 1 is likewise blocked by the particle on site 2. The particle on site $N$ also finds its movement thwarted by the particle on site 1 due to the periodic-boundary conditions.

fied for any given system, our method also provides a way to numerically calculate the nonuniversal scaling constants that, together with the known form of the DLSF, can be used to calculate properties such as the particle hopping distribution or the large deviation function.

This paper will begin with a short review of the continuous-time single-particle system from which Derrida et al. [29,30] initially derived the DLSF in Sec. II. Then, we will reveal a small extension of the results of Derrida et al. that will allow us to measure the DLSF for the discrete-time ASEP. Next, we describe our method and its use with the discrete-time single- and multiple-particle ASEP as an example of the application of our method. Last, we will discuss the applicability of this method to other systems of interest.

\section{REVIEW OF THE ASYMMETRIC EXCLUSION PROCESS}

Derrida et al. [29,30] examine a periodically bound continuous-time single-particle totally ASEP with $N$ sites each capable of holding one particle as shown in Fig. 1. These particles may move only to the right with a probability $d t$ if the target site is unoccupied. The total number of particles in the system is fixed at $p$.

In order to characterize the probability distribution $P\left(y_{t}\right)$ of the cumulative particle hopping per site $y_{t}$, they calculate the generating function $Z$, given by

$$
Z=\left\langle\exp \left[\gamma y_{t}\right]\right\rangle
$$

where the brackets $\langle\cdot\rangle$ denote the ensemble average. In order to solve for this generating function, they use the large time$t$ behavior [29],

$$
\left\langle\exp \left[\gamma y_{t}\right]\right\rangle \sim \exp \left[\lambda_{N}(\gamma) t\right]
$$

where $\lambda_{N}(\gamma)$ is the largest eigenvalue of the modified transfer matrix $\hat{T}$. If so inclined, the reader may refer to Appendix A to examine the full solution to $\lambda_{N}(\gamma)$, originally obtained by Derrida and Lebowitz in [29] and analytically continued by Derrida and Appert in [30]. The most important feature of this solution for $\lambda_{N}(\gamma)$ is that it has the scaling behavior

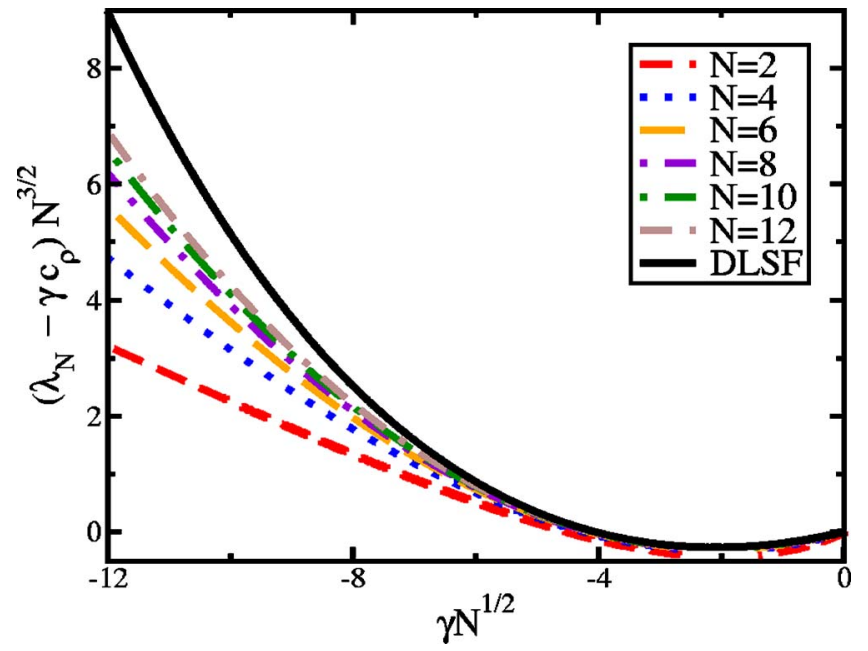

FIG. 2. (Color online) Plot of the left-hand side of Eq. (3) rescaled by $N^{3 / 2}$ versus $\gamma N^{1 / 2}$ for variously sized systems. A plot of the appropriately rescaled DLSF $G$ is included for reference. The curves for smaller $N$ do not agree well with the DLSF. This tells us that the left-hand side of Eq. (3) cannot be described by $G$ alone for small system sizes. The gap in the curves is a consequence of the dramatic increase in computational effort in its vicinity due to the slow convergence of the infinite series defining the solutions $\lambda_{N}(\gamma)$ close to their radii of convergence (see Appendix A).

$$
\lambda_{N}(\gamma)-\gamma c_{\rho}=\frac{a_{\rho} G\left(\gamma N^{1 / 2} b_{\rho}\right)}{N^{3 / 2}}
$$

for fixed filling ratio $p / N=\rho$ in the scaling limit $N \rightarrow \infty$ with $\gamma N^{1 / 2}$ held constant. $a_{\rho}, b_{\rho}$, and $c_{\rho}$ are all constants that depend solely on the particle density $\rho$. The most interesting aspect of this solution is the Derrida-Lebowitz scaling function $G$, whose form is independent of all system parameters. This led Derrida and Appert [30] to postulate that, in fact, $G$ represents a universal scaling function that can be used to describe all systems within the KPZ universality class. For details about the explicit representation of the Derrida-Lebowitz scaling function, the reader may refer to Appendix B.

\section{SCALING BEHAVIOR FOR INTERMEDIATE $N$}

Equation (3) contains a formula for successfully describing $\lambda_{N}(\gamma)$ in the scaling regime of constant $\gamma N^{1 / 2}$ in the limit of large system sizes. However, when considering small systems or large $\gamma$ at a fixed system size, Eq. (3) no longer adequately describes $\lambda_{N}(\gamma)$. Figure 2 shows just how poorly Eq. (3) performs under these conditions. If one wanted to extract the scaling function $G$ from numerical data for $\lambda_{N}(\gamma)$ in order to, e.g., verify its universality in a different system, one would have to go to very large system sizes $N$.

Here, we introduce another approach that can be utilized to directly verify the scaling behavior of $\lambda_{N}(\gamma)$. To this end, we exploit the fact that $\lambda_{N}(\gamma)$ not only has a well-defined scaling limit as $N \rightarrow \infty$ at constant $\gamma N^{1 / 2}$ (which implies $\gamma \rightarrow 0$ ) but that there is also a well-defined scaling limit as $N \rightarrow \infty$ at constant $\gamma$ for $\gamma<0$. This scaling limit is given [30] by 


$$
\lim _{N \rightarrow \infty} \lambda_{N}(\gamma) \equiv \lambda_{\infty}(\gamma)=\frac{-(1-\exp [\gamma \rho])\{1-\exp [\gamma(1-\rho)]\}}{1-\exp [\gamma]}
$$

This behavior is not universal, as the explicit dependence of the result on the particle density $\rho$ shows. However, the existence of such a scaling limit should still be a universal feature of all KPZ systems.

We propose that a combination of the two scaling limits in Eqs. (3) and (4) provides a faithful representation of the full function $\lambda_{N}(\gamma)$ for $\gamma<0$ and very small system sizes $N$. More precisely, we suggest that

$$
\lambda_{N}(\gamma)-\lambda_{\infty}(\gamma) \approx \frac{a_{\rho} G\left(\gamma N^{1 / 2} b_{\rho}\right)}{N^{3 / 2}}+\frac{a_{\rho}\left(\gamma b_{\rho}\right)^{3}}{24 \pi}
$$

already for very moderately sized $N=O(10)$. This agreement is essentially due to the fact that $\lambda_{\infty}(\gamma)$ provides an approximation of the $\lambda_{N}(\gamma)$ behavior that is far superior to the linear approximation from which the Derrida-Lebowitz scaling function $G$ was originally derived.

It is instructive to verify that our new relation (5) indeed simplifies to the two known scaling relations (3) and (4) in the appropriate limits. For fixed $\gamma<0$ the argument of the Derrida-Lebowitz scaling function $G$ on the right-hand side goes to negative infinity as $N \rightarrow \infty$. In this limit it is known [30] that

$$
G(\beta) \simeq-\frac{\beta^{3}}{24 \pi}=-\frac{\left(\gamma N^{1 / 2} b_{\Omega}\right)^{3}}{24 \pi}
$$

where the subleading terms decay exponentially with $|\beta|$. Since this leading term in $G$ cancels with the second term on the right-hand side of Eq. (5), the right-hand side vanishes as $N \rightarrow \infty$ and Eq. (4) emerges. If, on the other hand, $\gamma N^{1 / 2}$ is held constant as $N \rightarrow \infty, \gamma$ vanishes. Thus, the function $\lambda_{\infty}(\gamma)$ can be expanded for small $\gamma$. Since $\lambda_{\infty}(\gamma)$ is an odd function, this expansion contains only terms with odd powers of $\gamma$. The linear term precisely yields the term $\gamma c_{\rho}$ in Eq. (3). The $\gamma^{3}$ term equals the second term on the right-hand side of Eq. (5) [note that this implies a nontrivial connection between the nonuniversal constants $a_{\rho}, b_{\rho}$, and $c_{\rho}$ and the nonuniversal function $\lambda_{\infty}(\gamma)$ ]. All higher-order terms in $\gamma$ become subleading as $N \rightarrow \infty$ in this scaling limit. Thus, Eq. (3) reemerges. As becomes clear from this discussion, the additional term on the right-hand side of Eq. (11) can either be understood as a $\gamma^{3}$ correction to the left-hand side or as a correction to the DLSF $G$ on the right-hand side. It is the only term the scaling behavior of which allows it to be interpreted as both a part of the universal scaling function as well as a part of the infinite-size solution $\lambda_{\infty}(\gamma)$. For the purpose of this paper we will integrate this term into our scaling function and call the function

$$
\hat{G}(\beta) \equiv G(\beta)+\frac{\beta^{3}}{24 \pi}
$$

the adjusted Derrida-Lebowitz scaling function.

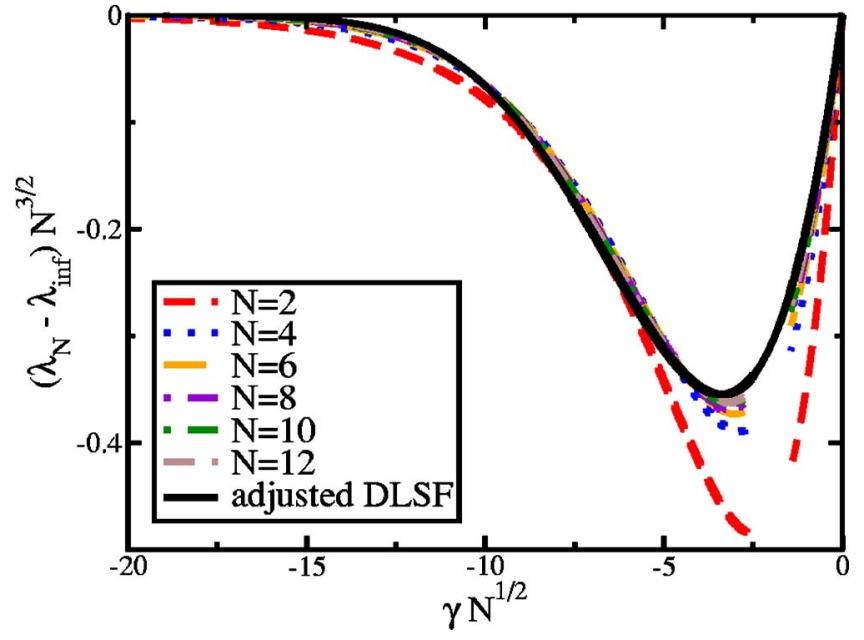

FIG. 3. (Color online) Plot of the left-hand side of Eq. (5), rescaled by $N^{3 / 2}$, for systems size $N=2,4,6,8,10$, and 12 . Notice how, even in the case of very small sizes, these values plotted are almost indistinguishable from not only each other, but the adjusted DLSF, given in Eq. (7) which they are plotted against. The gap in the data occurs due to the slow convergence of the plotted solutions (discussed in Appendix A) around the point $B_{c}$ [as given by Eq. (A3)].

Note that while Eq. (5) has only been shown to hold in the two limiting cases discussed above, in actuality, it works exceedingly well even for small systems. Figure 3 shows the left-hand side of Eq. (5), rescaled by $N^{3 / 2}$, plotted for $N=2$, $4,6,8,10$, and 12. The right-hand side of Eq. (5) is plotted as well, with the appropriate rescaling factors given by [30]. The quality with which Eq. (5) captures the behavior of this intermediate-scaling region is suprising, especially when considering the small system sizes. This empirical observation forms the foundation of the methodology presented herein for numerically understanding the behavior of quantities, such as particle hopping, in systems that currently remain beyond the reach of purely analytic methods.

\section{DISCRETE-TIME ASYMMETRIC EXCLUSION PROCESS}

In the following, as a specific application of our method, we verify the universality of the DLSF for the discrete-time totally ASEP using the sublattice-parallel updating scheme (see Fig. 4) [13]. In our totally ASEP, we consider a periodically bound system of size $N$ where $p$ particles can only move to the right and each site can accommodate up to $n$ particles. During each odd-time interval $t$, the positions with odd numbers are evaluated for transitions. Particles can hop only if available space exists to the right for the particle to move-i.e., only if the site does not already contain the maximum number of allowed particles, $n$. For allowed transitions, particles hop with probability $\eta$ and stay put with probability $1-\eta$. If there is more than one particle on a site, only one particle is considered for hopping. For even-time intervals, the exact same dynamic occurs at the evennumbered positions. Notice that, in order to use this 


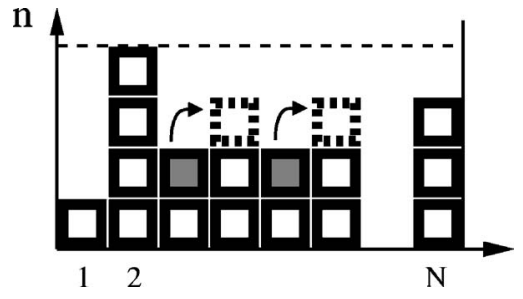

FIG. 4. Diagram of our discrete-time asymmetric exclusion process. The diagram shows an odd time where only particles on even sites are considered for hopping. Notice that the particle at site 1 cannot move because site 2 is already at maximum occupancy. However, for sites 3 and 5, the shaded particles can hop to the right with a probability $\eta$, as shown by the boxes with dotted outlines.

sublattice-parallel updating scheme, the number of sites, $N$, must be even. For this system, we solve via a transfer matrix method described in Appendix $\mathrm{C}$ for the exponential term $\lambda_{N}$ of the generating function $Z$ of the total particle flux per site, $y_{t}$, in the large-time limit $t \rightarrow \infty$. Again, let us first define this generating function as

$$
Z_{t, N}(\gamma ; \Omega) \equiv\left\langle\exp \left[\gamma y_{t}\right]\right\rangle
$$

where the brackets $\langle\cdot\rangle$ denote the ensemble average and $\Omega$ summarizes the specific parameters contributing to the evaluation of the particle flux per site $y_{t}$-i.e., $\eta, p$, and $n$. As in the continuous-time case examined by Derrida and Lebowitz [29], the generating function behaves like

$$
Z_{t, N}(\gamma ; \Omega) \sim \exp \left[\lambda_{N}(\gamma ; \Omega) t\right]
$$

for large times, where $\lambda_{N}(\gamma ; \Omega)$ is the largest eigenvalue of the characteristic matrix $\hat{T}_{N}(\gamma ; \Omega)$, the technical details of whose construction are provided in Appendix C.

Since we will only be able to numerically evaluate $\lambda_{N}(\gamma ; \Omega)$ for relatively small $N$, we apply the method presented in the previous section. Toward this end, we introduce the function $\lambda_{\infty}(\gamma ; \Omega)$ describing the infinite-size behavior of the $\lambda_{N}(\gamma ; \Omega)$ as

$$
\lambda_{\infty}(\gamma ; \Omega)=\lim _{N \rightarrow \infty} \lambda_{N}(\gamma ; \Omega) .
$$

The new scaling form then becomes

$$
\lambda_{N}(\gamma ; \Omega)-\lambda_{\infty}(\gamma ; \Omega)=\frac{a_{\Omega} \hat{G}\left(\gamma N^{1 / 2} b_{\Omega}\right)}{N^{3 / 2}} .
$$

For the specific case in which the allowed number of particles per site equals $1, n=1$, and the system is half filled, $p=N / 2$, there exists an exact analytical solution $[16,17]$ for $\lambda_{\infty}(\gamma ; \Omega)$ :

$$
\lambda_{\infty}\left(\gamma ; \Omega_{n=1, p=N / 2}\right)=\ln \left(\frac{\sqrt{\eta}+\exp [-\gamma]}{1+\sqrt{\eta} \exp [-\gamma]}\right) .
$$

This, combined with our numerical transfer matrix method for calculating $\lambda_{N}(\gamma ; \Omega)$, allows us to directly measure the left-hand side of Eq. (11). Since the adjusted DLSF on the right-hand side has already been solved, only the scaling coefficients $a_{\Omega}$ and $b_{\Omega}$ are unknown. This allows us to nu-

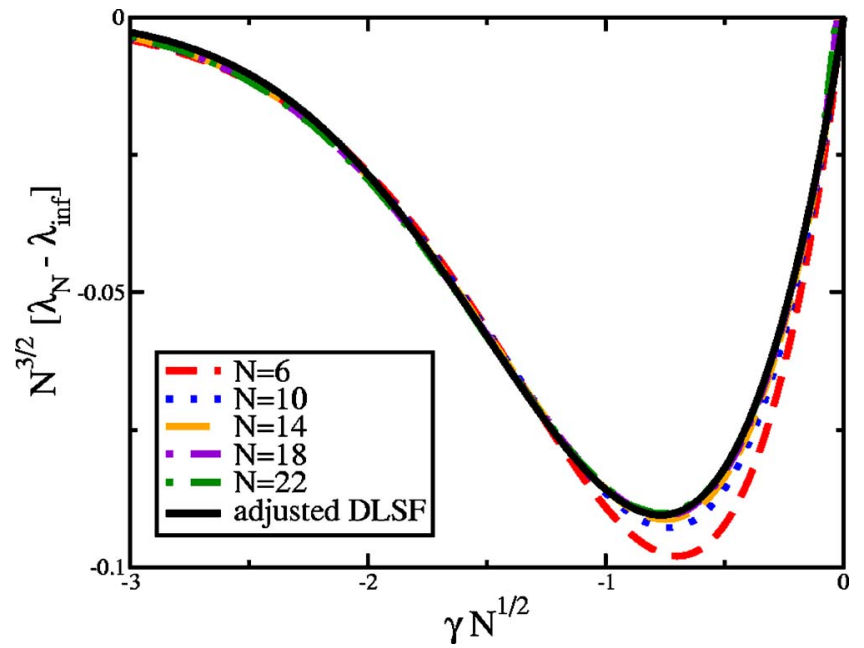

FIG. 5. (Color online) The appropriately rescaled and adjusted DLSF [in accordance with the right-hand side of Eq. (13)] plotted against the direct measurement of the left-hand side of Eq. (13) for $n=1, \rho=p /(n N)=1 / 2$, and $\eta=3 / 4$. The left-hand side of Eq. (11) has been plotted for $N=6,10,14,18$, and 22. It becomes difficult to distinguish the values for the larger widths from the adjusted DLSF solved in [30] since they lie in near-perfect agreement. This indicates that the proposed universality of the DLSF does hold for this system. The scaling factors $a_{\Omega}$ and $b_{\Omega}$ in Eq. (11), neither of which depends on the finite-size effects, are chosen only once for the largest system, $N=22$, and control the scaling of the adjusted DLSF.

merically calculate the left-hand side of Eq. (11) and use the results to fit $a_{\Omega}$ and $b_{\Omega}$ for one (the largest) system size $N$. For convenience, here we multiply Eq. (11) by $N^{3 / 2}$,

$$
N^{3 / 2}\left[\lambda_{N}(\gamma ; \Omega)-\lambda_{\infty}(\gamma ; \Omega)\right]=a_{\Omega} \hat{G}\left(\gamma N^{1 / 2} b_{\Omega}\right),
$$

and plot the left-hand side of Eq. (13) against $\beta \equiv \gamma N^{1 / 2}$ in Fig. 5 for $N=6,10,14,18$, and 22 . The agreement between the various curves in Fig. 5 gives a good indication that the asymptotic solution for the DLSF indeed applies to our discrete time ASEP.

\section{DISCRETE-TIME MULTIPARTICLE ASYMMETRIC EXCLUSION PROCESS}

In order to give a further example of the usability of our method, we also apply it to the case where we allow more than one particle per site. A lattice point with more than one allowed state or highway traffic with more than one lane both provide very good examples of why such a multipleparticle ASEP is important in and of itself. For $n>1$, an analytic solution for $\lambda_{\infty}(\gamma ; \Omega)$ does not exist. Thus, we cannot quite follow the method shown in the previous sections. However, we can still test both the validity of the DLSF and calculate the nonuniversal scaling factors through other means. Since we have a method for obtaining $\lambda_{N}(\gamma ; \Omega)$, we take the difference 


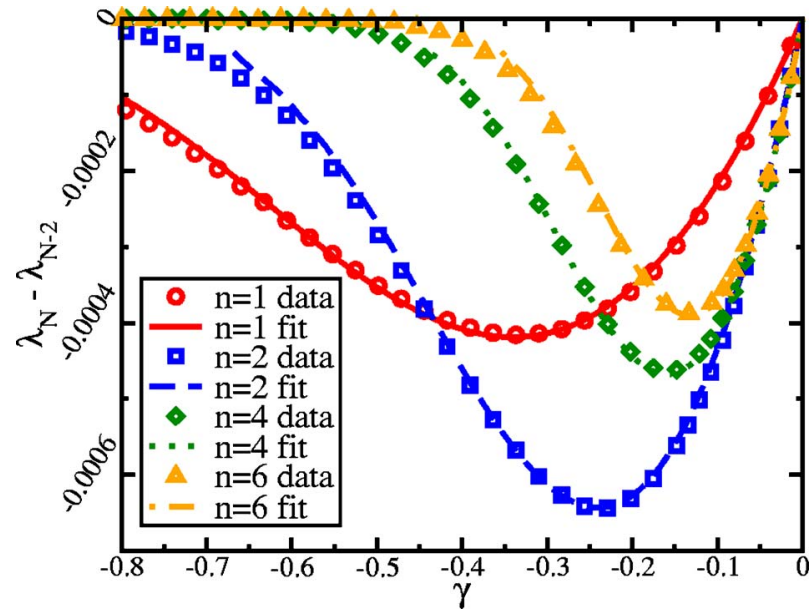

FIG. 6. (Color online) Plot of the right-hand side of Eq. (14) versus the appropriately fitted left-hand side for $\eta=3 / 4$ for various $N$. This plots shows the excellent agreement between the calculated data and the proposed form of Derrida et al. of the scaling function under different parameter choices. The total number of particles has been chosen such that $p=N n / 2$-i.e., a half-filled system. For $n=1, N=22$ was used, for $n=2, N=14$, for $n=4, N=10$, and for $n=6, N=8$.

$$
\begin{aligned}
\lambda_{N}(\gamma ; \Omega)-\lambda_{N-2}(\gamma ; \Omega)= & \frac{a_{\Omega} G\left(\gamma N^{1 / 2} b_{\Omega}\right)}{N^{3 / 2}} \\
& -\frac{a_{\Omega} G\left(\gamma(N-2)^{1 / 2} b_{\Omega}\right)}{(N-2)^{3 / 2}}
\end{aligned}
$$

in order to eliminate the need for the value of $\lambda_{\infty}(\gamma ; \Omega)$. Notice that the size-independent terms $\left[a_{\Omega}\left(\gamma b_{\Omega}\right)^{3} /(24 \pi)\right]$ cancel out, leaving us with the original DLSF $G$. Once again, we find the scaling coefficients $a_{\Omega}$ and $b_{\Omega}$ by fitting the righthand side to the left-hand side of Eq. (14) for a single $N$. Figure 6 shows the right-hand side of Eq. (14) for the largest $N$ with the numerically fitted values for $a_{\Omega}$ and $b_{\Omega}$ plotted against the left-hand side whose value is obtained using the same transfer matrix method described in Appendix C. While in this approach curves obtained for different $N$ do not overlap each other, we note that we obtain similarly good agreement as in Fig. 6 for small system sizes $N$ without refitting the scaling parameters $a_{\Omega}$ and $b_{\Omega}$ (data not shown). This once again results in excellent agreement between our solution for the left-hand side and the properly rescaled righthand side for various parameter values $\Omega$ including different values for $n, \eta$, and $N$.

Figures 5 and 6 each display excellent agreement with established KPZ theory and come without any real adjustment or reformulation of existing theory. They are simply the direct result of studying our multiparticle discrete-time ASEP dynamics modeled by a transfer matrix method. Once $a_{\Omega}$ and $b_{\Omega}$ have been determined, their values can be utilized in order to numerically compute the infinite form $\lambda_{\infty}(\gamma ; \Omega)$ from $\lambda_{N}(\gamma ; \Omega)$ for even small system sizes by utilizing Eq. (11) - a fact which is particularly important in cases where $\lambda_{\infty}(\gamma ; \Omega)$ remains unsolved. Of course, given a form for $\lambda_{\infty}(\gamma ; \Omega)$, even though it be numerical, allows one to calculate proper- ties of the system in the thermodynamic limit, such as the particle hopping and large deviation function.

In summary, the fast convergence in $N$ allows for rapid calculation of an entire function $\lambda_{\infty}(\gamma ; \Omega)$, an otherwise difficult quantity to compute for most systems. The accessibility of $\lambda_{\infty}(\gamma ; \Omega)$ through studies of smaller systems has already been put to use in calculating practical quantities important in sequence alignment [18].

\section{CONCLUSION}

In this paper, we have presented a numerical method that allows a direct numerical calculation of the universal DLSF and its nonuniversal scaling factors. In contrast to previous numerical studies, our method does not rely on sampling but rather on the exact diagonalization of sparse modified transfer matrices for very small finite-size systems. Thus, it is able to capture the full information on rare events that the DLSF encodes. As an application, we have extended the universality of the Derrida-Lebowitz scaling function to inherently discrete-time hopping processes with multiple particles per site by directly measuring the scaling function itself. We found that the numerically determined scaling function converges toward the universal scaling function already for relatively small finite systems.

The method we have outlined is applicable to a variety of discrete KPZ systems, including the important openboundary ASEP and the open-boundary partially ASEP used for modeling true nonequilibrium-driven lattice-gas dynamics. In addition to this, the more complex interactions of multiple number and types of particles can be modeled using our method.

\section{ACKNOWLEDGMENTS}

R.B. gratefully acknowledges funding from the National Science Foundation through Grant Nos. DBI-0317335 and DMR-0404615.

\section{APPENDIX A: $\lambda_{N}$ FOR A CONTINUOUS-TIME TOTALLY ASYMMETRIC EXCLUSION PROCESS}

The solution for $\lambda_{N}(\gamma)$ can be given by the parametric equation [29]

$$
\begin{aligned}
\lambda_{N}(\gamma) & =-p \sum_{q=1}^{\infty} B^{q} \frac{(N q-2) !}{(p q) !(N q-p q-1) !}, \\
\gamma & =-N \sum_{q=1}^{\infty} B^{q} \frac{(N q-1) !}{(p q) !(N q-p q) !},
\end{aligned}
$$

for $-B_{c}<B<B_{c}$, where the radius of convergence $B_{c}$ is given by

$$
B_{c}=\frac{p^{p}(N-p)^{(N-p)}}{N^{N}}
$$

This set of equations represents the solution for $\lambda_{N}(\gamma)$ in the region $\gamma_{-}<\gamma<\gamma_{+}$, where 


$$
\begin{gathered}
\gamma_{-}=-N \sum_{q=1}^{\infty}\left(B_{c}\right)^{q} \frac{(N q-1) !}{(p q) !(N q-p q) !} \\
\gamma_{+}=-N \sum_{q=1}^{\infty}\left(-B_{c}\right)^{q} \frac{(N q-1) !}{(p q) !(N q-p q) !} .
\end{gathered}
$$

Derrida and Appert term this the "scaling region"-i.e., the region where Eq. (3) holds true in the limit $N \rightarrow \infty$.

In the strongly negative region $\gamma<\gamma_{-}$, the solution is given by the analytic continuation of Eqs. (A1) and (A2), found in [30],

$$
\begin{gathered}
\lambda_{N}(\gamma)=\frac{\frac{1}{2 B^{1 / p}}\left(1-2 B^{1 / p}-\sqrt{1-4 B^{1 / p}}\right)}{1+\frac{1}{2 B^{1 / p}}\left(1-2 B^{1 / p}-\sqrt{1-4 B^{1 / p}}\right)} \\
-\frac{\frac{1}{2 B^{1 / p}}\left(1-2 B^{1 / p}+\sqrt{1-4 B^{1 / p}}\right)}{1+\frac{1}{2 B^{1 / p}}\left(1-2 B^{1 / p}+\sqrt{1-4 B^{1 / p}}\right)} \\
-p \frac{\infty}{q=1} \frac{(N q-2) !}{(p q) !(N q-p q-1) !}, \\
-\frac{\ln \left(1+\frac{1}{2 B^{1 / p}}\left(1-2 B^{1 / p}-\sqrt{1-4 B^{1 / p}}\right)\right.}{\ln \left(1+\frac{1}{2 B^{1 / p}}\left(1-2 B^{1 / p}+\sqrt{1-4 B^{1 / p}}\right)\right)} \\
-N \sum_{q=1}^{\infty} B^{q} \frac{(N q-1) !}{(p q) !(N q-p q) !},
\end{gathered}
$$

for $0<B<B_{c}$.

\section{APPENDIX B: THE DERRIDA-LEBOWITZ SCALING FUNCTION}

The form of the Derrida-Lebowitz scaling function can be given as

$$
\begin{gathered}
G(\beta)=\frac{4}{3 \sqrt{\pi}} \int_{0}^{\infty} \epsilon^{3 / 2} \frac{C e^{-\epsilon} d \epsilon}{1+C e^{-\epsilon}}, \\
\beta=\frac{2}{\sqrt{\pi}} \int_{0}^{\infty} \epsilon^{1 / 2} \frac{C e^{-\epsilon} d \epsilon}{1+C e^{-\epsilon}},
\end{gathered}
$$

for $C>-1$. For the region less than $\beta_{-} \equiv \lim _{C \rightarrow-1} \beta$, the analytic continuation of $G$ is given by

$$
\begin{gathered}
G(\beta)=\frac{8}{3} \sqrt{\pi}[-\ln (-C)]^{3 / 2}-\sum_{q=1}^{\infty}(-C)^{q} q^{-5 / 2}, \\
\beta=-4 \sqrt{\pi}[-\ln (-C)]^{1 / 2}-\sum_{q=1}^{\infty}(-C)^{q} q^{-3 / 2},
\end{gathered}
$$

for $0<C<1$.

\section{APPENDIX C: BRIEF DESCRIPTION OF THE MATRIX METHOD}

We shortly describe how we actually calculate $\lambda_{N}(\gamma ; \Omega)$. In our brand of the ASEP, we use a sublattice parallel updating scheme where hopping probabilities in even- and oddtime intervals are evaluated separately. Because our discrete time processes may be thought of as a combination of a number of very simple processes occurring in some sequential order, we may first examine the base dynamic and expand this into the larger picture. Before examining the movement at all positions, we study the dynamics of a single hopping transition. We first create the transfer matrix $T(\gamma=0 ; \Omega)$ describing the transition probabilities for one pair of sites [17] using particle occupancy number $d$ as our basis. Next, we modify this matrix by multiplying all off-diagonal elements by the factor $\exp [-\gamma / N]$. This effectively tags the average number of hops per site. For $n=2$ particles per site this results in the matrix

$$
T\left(\gamma ; \Omega_{n=2}\right)=\left(\begin{array}{ccccccccc}
1 & 0 & 0 & 0 & 0 & 0 & 0 & 0 & 0 \\
0 & \eta & 0 & 0 & 0 & 0 & 0 & 0 & 0 \\
0 & 0 & \eta & 0 & 0 & 0 & 0 & 0 & 0 \\
0 & z & 0 & 1 & 0 & 0 & 0 & 0 & 0 \\
0 & 0 & z & 0 & \eta & 0 & 0 & 0 & 0 \\
0 & 0 & 0 & 0 & 0 & \eta & 0 & 0 & 0 \\
0 & 0 & 0 & 0 & z & 0 & 1 & 0 & 0 \\
0 & 0 & 0 & 0 & 0 & z & 0 & 1 & 0 \\
0 & 0 & 0 & 0 & 0 & 0 & 0 & 0 & 1
\end{array}\right)
$$

in the basis $(00,01,02,10,11,12,20,21,22)$ where $z=(1-\eta) \exp [-\gamma / N]$.

From this smaller transfer matrix, we may build up this single-pair description into the larger- $N$-site picture by taking the tensor product

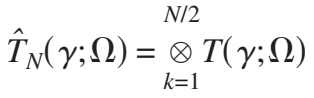

and eliminating any states that do not contain the right number of particles, $p$. This gives us the matrix that models particle hopping from sites in one time interval. Converting this to the appropriate basis for the next time interval can be done by utilizing the translation operator $C$ defined such that

$$
C\left|d_{0} d_{1} \cdots d_{N-1}\right\rangle \equiv\left|d_{1} d_{2} \cdots d_{N-1} d_{0}\right\rangle
$$

After exploiting translational invariance and up-down mirror symmetry in order to reduce the size of our state space we obtain the additional identity $C=C^{-1}$ on this reduced state space. Then the matrix product $\hat{T}_{\text {even }} \hat{T}_{\text {odd }}=\hat{T}_{N}\left(C^{-1} \hat{T}_{N} C\right)$ $=\left(\hat{T}_{N} C\right)^{2}$ describes the particle hopping of our discrete time ASEP for all $N$ sites. These dynamics can be viewed as a Markov process on a $(n+1)^{N}$-dimensional state space of the equal-time difference vector $|d(0, t), d(1, t), \ldots, d(N, t)\rangle$. Solving for the largest eigenvalue $\xi_{N}(\gamma ; \Omega)$ of $\hat{T}_{N} C$ gives us the particle hopping function for finite size $[16,17]$ 


$$
\lambda_{N}(\gamma ; \Omega)=\ln \xi_{N}(\gamma ; \Omega) .
$$

This is the function used in order to produce the results given above. Calculating this largest eigenvalue is somewhat challenging, since this matrix description grows very quickly with $N$. However, the matrices are very sparse with the num- ber of nonzero matrix elements growing almost linearly with the matrix dimension. This makes it possible to numerically obtain the largest eigenvalue required in Eq. (C4) using the implicitly restarted Arnoldi method [34] for matrix dimensions up to around $10^{5}$. This allows us to plot the entire function $\lambda_{N}(\gamma ; \Omega)$ in very reasonable time scales.
[1] M. Kardar, G. Parisi, and Y. C. Zhang, Phys. Rev. Lett. 56, 889 (1986)

[2] D. Kandel, E. Domany, and B. Nienhuis, J. Phys. A 23, L755 (1990).

[3] J. Krug and H. Spohn, in Solids far from Equilibrium, edited by C. Godréche (Cambridge University Press, Cambridge, England, 1991).

[4] E. Brunet and B. Derrida, Phys. Rev. E 61, 6789 (2000).

[5] R. Bundschuh and M. Lässig, Phys. Rev. E 54, 304 (1996).

[6] M. Lässig and H. Kinzelbach, Phys. Rev. Lett. 78, 903 (1997).

[7] A. M. Povolotsky, V. B. Priezzhev, and Chin-Kun Hu, Physica A 321, 280 (2003).

[8] C.-C. Chen and M. den Nijs, Phys. Rev. E 65, 031309 (2002); 66, 011306 (2002).

[9] T. Haplin-Healy and Y. Zhang, Phys. Rep. 254, 215 (1995).

[10] J. Krug, Adv. Phys. 46, 139 (1997).

[11] P. Meakin, Phys. Rep. 235, 189 (1993).

[12] H. Spohn, Large Scale Dynamics of Interacting Particles (Springer, Berlin, 1991).

[13] N. Rajewsky, L. Santen, A. Schadschneider, and M. Schreckenberg, J. Stat. Phys. 92, 151 (1999).

[14] V. Belitsky, J. Krug, E. Neves, E. Jordão, and G. M. Schütz, J. Stat. Phys. 103, 945 (2001).

[15] L. B. Shaw, R. K. P. Zia, and K. H. Lee, Phys. Rev. E 68, 021910 (2003).

[16] R. Bundschuh, in Proceedings of the Fourth Annual International Conference on Computational Molecular Biology (RECOMB2000), edited by S. Istrail et al. (ACM Press, New York, 2002), p. 86.

[17] R. Bundschuh, Phys. Rev. E 65, 031911 (2002).
[18] N. Chia and R. Bundschuh, Proceedings of the Ninth Annual International Conference on Computational Molecular Biology (RECOMB2005), edited by S. Miyano et al. (SpringerVerlag, Berlin, 2005), p. 474.

[19] B. Derrida, Phys. Rep. 301, 65 (1998).

[20] B. Derrida, C. Enaud, and J. L. Lebowitz, J. Stat. Phys. 115, 365 (2004).

[21] B. Derrida, M. R. Evans, V. Hakim, and V. Pasquier, J. Phys. A 26, 1493 (1993).

[22] D. Dhar, Phase Transitions 9, 51 (1987).

[23] L. H. Gwa and H. Spohn, Phys. Rev. Lett. 68, 725 (1992); Phys. Rev. A 46, 844 (1992).

[24] S. A. Janowsky and J. L. Lebowitz, Phys. Rev. A 45, 618 (1992).

[25] J. Krug, Phys. Rev. Lett. 67, 1882 (1991).

[26] P. Meakin, P. Ramanlal, L. M. Sander, and R. C. Ball, Phys. Rev. A 34, 5091 (1986).

[27] D. Kim, Phys. Rev. E 52, 3512 (1995).

[28] R. B. Stinchcombe and G. M. Schütz, Europhys. Lett. 29, 663 (1995); Phys. Rev. Lett. 75, 140 (1995).

[29] B. Derrida and J. L. Lebowitz, Phys. Rev. Lett. 80, 209 (1998).

[30] B. Derrida and C. Appert, J. Stat. Phys. 94, 1 (1999).

[31] D. S. Lee and D. Kim, Phys. Rev. E 59, 6476 (1999).

[32] C. Appert, Phys. Rev. E 61, 2092 (2000).

[33] I. Weichsel, Int. J. Mod. Phys. C 11, 691 (2000).

[34] R. B. Lehoucq, D. C. Sorensen, and C. Yang, ARPACK Users' Guide: Solutions of Large Scale Eigenvalue Problems with Implicitly Restarted Arnoldi Methods (SIAM, Philadelphia, 1997). 